\title{
Improved Wettability and Dimensional Stability of Bamboo Timber by Coating Graphene/Silica Composites
}

\author{
Jin Wang $\mathbb{D},{ }^{1}$ Ye Zhou, ${ }^{2}$ Jingpeng Li $\mathbb{D},{ }^{3}$ Yongshun Feng, ${ }^{1}$ Jian Zhang, ${ }^{1}$ Haixia Yu $\mathbb{D},{ }^{1}$ \\ and Xiaowei Zhuang ${ }^{1}$ \\ ${ }^{1}$ Zhejiang Provincial Key Lab of Biological and Chemical Utilizing of Forest Resources, Zhejiang Forestry Academy, \\ Hangzhou 310023, China \\ ${ }^{2}$ College of Environment, Zhejiang University of Technology, Hangzhou 310023, China \\ ${ }^{3}$ Key Laboratory of High Efficient Processing of Bamboo of Zhejiang Province, China National Bamboo Research Center, \\ Hangzhou 310012, China
}

Correspondence should be addressed to Jingpeng Li; lijp@caf.ac.cn, Haixia Yu; ivyyhx@126.com, and Xiaowei Zhuang; zhuang413@sina.com

Received 3 June 2021; Accepted 30 July 2021; Published 17 August 2021

Academic Editor: Alenka Vesel

Copyright ( 2021 Jin Wang et al. This is an open access article distributed under the Creative Commons Attribution License, which permits unrestricted use, distribution, and reproduction in any medium, provided the original work is properly cited.

\begin{abstract}
Bamboo timber (BT) is a kind of natural porous material, and usually bamboo cracking and deformation are caused by the change of humidity in the environment. Inspired by the natural structure of the shell, a multilayer structure was designed to fabricate a kind of graphene/silica composite coating on the BT surface. The multilayer bamboo composite (FAS-RGO@SiO ${ }_{2} \mathrm{BT}$ ) was prepared by using the self-assembly process of nanospherical silica on graphene followed by hydrophobic modification. A possible formation mechanism of FAS-RGO@SiO $\mathrm{ST}_{2} \mathrm{BT}$ was discussed by X-ray diffraction, scanning electron microscopy, energy-dispersive X-ray analysis, X-ray photoelectron spectroscopy, and Fourier transform infrared spectroscopy. The FAS-RGO@SiO ${ }_{2} \mathrm{BT}_{\text {has }}$ excellent hydrophobic properties for tea, orange juice, milk, coffee, blue ink, red wine, and green juice. Also, the FAS-RGO@ SiO 2 BT has an obvious hydrophobic effect on any of the various solutions with a $\mathrm{pH}$ of 1 to 14 . In addition, after a 90-day immersion test, the water absorption rate of FAS-RGO@ $\mathrm{SiO}_{2} \mathrm{BT}$ is $9.7 \%$ and the thickness swelling rate is $0.5 \%$. The wettability and dimensional stability of the FAS- $\mathrm{RGO} @ \mathrm{SiO}_{2} \mathrm{BT}$ are significantly improved compared to those of BT.
\end{abstract}

\section{Introduction}

Bamboo is a kind of natural porous material and has different pores, such as cell cavity and microfilament space. Moreover, the bamboo chemical composition contains lots of hydrophilic groups. Water absorption and fiber swelling result from the combination of bamboo's moisture and chemical components via the carbon-carbon bond or hydrogen bond. The size changes of bamboo products are often caused by the change of humidity in the environment, resulting in further cracking and deformation [1]. Thus, it is necessary to study waterproof treatment methods for bamboo to improve dimensional stability. The common methods are usually oil painting, waxy coating, and high-temperature treatment [2]. Recently, a large number of studies have been devoted to the study of hydrophobic treatment of bamboo or wood. Seo et al. reported that stable and water droplet shockresistant superhydrophobic surfaces can be rapidly prepared using only a single candle [3]. The fabrication process is very simple and does not require additional solvents. Wang et al. used the sol-gel method to deposit $\mathrm{SiO}_{2}$ particles on the surface of the wood. After vapor deposition of $1 \mathrm{H}-, 2 \mathrm{H}-$, and 2H-perfluoroalkyl trimethoxysilane (POTS), superhydrophobic wood was obtained [4]. The water contact angle on the wood surface was $164^{\circ}$, and the rolling angle was less than $3^{\circ}$. A series of studies [5-9] on the preparation of superhydrophobic wood by a hydrothermal method and lowtemperature hydrothermal method have been conducted. Nanoparticles, such as $\mathrm{FeOOH}, \mathrm{TiO}_{2}, \mathrm{Cu}_{2} \mathrm{O}, \mathrm{CoFe}_{2} \mathrm{O}_{4}$, and $\mathrm{WO}_{3}$, were synthesized on the wood surface by the 
hydrothermal method and then modified with low surface energy using hydrophobic silane reagents and fluorinecontaining reagents. The prepared coatings have excellent superhydrophobic properties, high temperature and humidity, light radiation, and wear resistance. Lozhechnikova et al. used the electrophoresis process to assemble carnauba wax particles and $\mathrm{ZnO}$ nanoparticles on the wood surface to form a protective coating. The coating has unique superhydrophobicity and certain ultraviolet protection ability [10]. Li et al. used several media treatments on bamboo scriber and found that the oil heat-treated group had higher dimensional stability [11].

Inspired by the strong adhesion ability of natural marine mussels, bionic mussel surface modification technology has gained widespread attention as a green, mild, efficient, and sustainable method [12]. Inspired by the natural structure of the shell, a multilayer structure was designed to fabricate a kind of superhydrophobic coating on the bamboo surface [13]. The BT was set as the bottom layer, the graphene sheets were set as the middle layer, and the surface layer was synthesized silicon dioxide nanoparticles. The composite material with three-layer structure was prepared by two-step hydrothermal treatment and further hydrophobic agent modification. The performance test shows that the as-prepared bamboo samples have excellent superhydrophobic performance and improved dimensional stability.

\section{Materials and Methods}

2.1. Materials. Graphite powder $(<20 \mu \mathrm{m})$ was supplied by Boyles Chemical Company (Shanghai). Sulfuric acid and nitric acid were supplied by Liudongshan Chemical Company (Lanxi). Methylene blue, methyl orange, rhodamine B, potassium permanganate, hydrogen peroxide, and ethanol were supplied by Kelong Chemical Company (Chengdu). The tetraethylorthosilicate (TEOS) was supplied by Bodi Chemical Company (Tianjin). Hydrochloric acid, ammonia, and (heptadecafluoro-1,1,2,2-tetradecyl)trimethoxysilane (FAS-17) were supplied by Huipu Chemical Company (Hangzhou). The Phyllostachys pubescens bamboo of 5 years was collected from Anji County, Zhejiang Province. The bamboo specimens were processed into blocks with dimensions of $50 \mathrm{~mm} \times 20 \mathrm{~mm} \times 5 \mathrm{~mm}$ and $20 \mathrm{~mm} \times 20 \mathrm{~mm} \times 5$ $\mathrm{mm}$ (longitudinal $\times$ chordwise $\times$ radial). These bamboo specimens were then cleaned by washing sequentially with deionized water and then ethanol for $30 \mathrm{~min}$ and were finally dried under vacuum at $50^{\circ} \mathrm{C}$ for $24 \mathrm{~h}$. The original bamboo timber subjected to the above process was abbreviated as BT.

2.2. Preparation of RGO@SiO $\mathrm{S}_{2} \mathrm{BT}$ and FAS-RGO@SiO${ }_{2} \mathrm{BT}$. The bamboo block with a reduced graphene oxide loading layer, which was abbreviated as RGOBT, was fabricated according to the previous method [14]. Then, $85 \mathrm{ml}$ of absolute ethanol, $9 \mathrm{ml}$ of deionized water, and $1.5 \mathrm{ml}$ of ammonia were added to a clean beaker and uniformly mixed under magnetic stirring. In the stirring process, $4.5 \mathrm{ml}$ ethyl orthosilicate was added slowly, which will make the colloid yellowish. The prepared RGOBT samples and the solution were placed in a Teflon-lined stainless steel autoclave at a specific volume ratio. The autoclave was sealed and kept at $110^{\circ} \mathrm{C}$ for $3 \mathrm{~h}$. After the hydrothermal reaction, the treated bamboo samples were removed and rinsed with deionized water and then dried at $50^{\circ} \mathrm{C}$ for $24 \mathrm{~h}$ in a vacuum. The treated bamboo with a silica/graphene coating was abbreviated as $\mathrm{RGO} @ \mathrm{SiO}_{2} \mathrm{BT}$.

To reduce the surface free energy and achieve a superhydrophobic surface, the as-prepared RGO@SiO 2 BT was further modified with FAS-17. The FAS-17 and methanol were mixed according to $1.0 \mathrm{wt}$ \% and stirred evenly. The RGO@$\mathrm{SiO}_{2} \mathrm{BT}$ was immersed into the mixed liquid and after magnetic stirring $24 \mathrm{~h}$ at room temperature $\left(20^{\circ} \mathrm{C}\right)$, and then, the sample was removed. The surfaces were washed with methanol, and the samples were dried at $50^{\circ} \mathrm{C}$ for $24 \mathrm{~h}$ in a vacuum. The above-treated $\mathrm{BT}$ was abbreviated as FASRGO@SiO 2 BT.

2.3. Characterization. The surface morphologies of the samples were characterized using scanning electron microscopy (SEM, Quanta 200, FEI, USA). The surface chemical compositions of the samples were determined via energy-dispersive spectroscopy (EDX, attached to the SEM). The crystalline structures of the samples were identified by X-ray diffraction (XRD, D/MAX 2200, Rigaku, Japan) using $\mathrm{Cu} \mathrm{K}_{\alpha}$ radiation $(\lambda=1.5418 \AA)$ at a $2 \theta$ scan rate of $4^{\circ} \cdot \mathrm{min}^{-1}, 40 \mathrm{kV}$, and $40 \mathrm{~mA}$, ranging from $5^{\circ}$ to $80^{\circ}$. The presence of functional groups in the samples was confirmed through Fourier transform infrared (FTIR) spectroscopy (Spectrum One, PerkinElmer, USA). The surface elemental composition analyses were performed based on the X-ray photoelectron spectroscopy (XPS, Thermo Fisher Scientific-K-Alpha 1063, UK) with an $\mathrm{Al} \mathrm{Ka}$ monochromatic X-ray source, in which all binding energies were calibrated regarding the $\mathrm{C}$ 1s peak $(284.8 \mathrm{eV})$.

2.4. Wettability Test. The water contact angle of BT, RGOBT, RGO@SiO 2 BT, and RGO@SiO 2 BT samples was measured using a contact angle tester (SL200KB). At room temperature, the liquid was $5 \mu \mathrm{l}$ distilled water, and the water contact angle was the average value of the five measurements. To test the hydrophobic effect of the sample surface, these eight kinds of liquid were collected, such as tea, orange juice, milk, coffee, edible oil, blue ink, red wine, and green fruit juice. Also, the hydrochloric acid and sodium hydroxide were used to test the hydrophobic effect of the bamboo surface for each $\mathrm{pH}$ solution in the range of 1-14. Finally, the prepared sample was subjected to a water boiling test and a long-term placement to test the hydrophobic effect.

2.5. Dimensional Stability Test. According to the relevant provision test method in GB/T 1928-2009 "General rules for wood physical and mechanical test methods," the dimensional stability of the samples was measured [15]. The size of the bamboo block was $20 \mathrm{~mm} \times 20 \mathrm{~mm} \times 5 \mathrm{~mm}$, and the number of samples was 15 for each test. The water absorption and thickness swelling rate were performed according to the above method. 


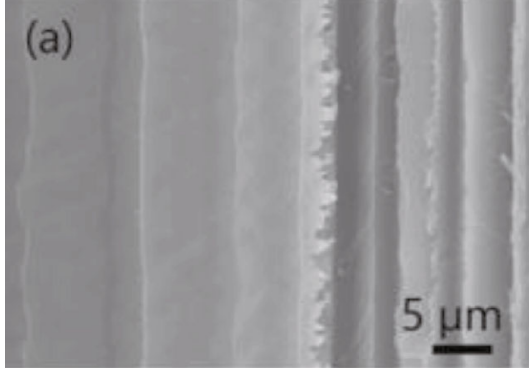

(a)

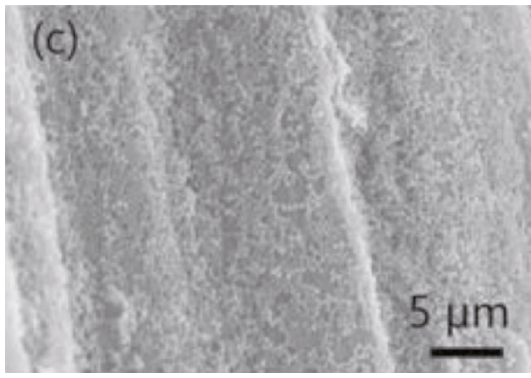

(c)

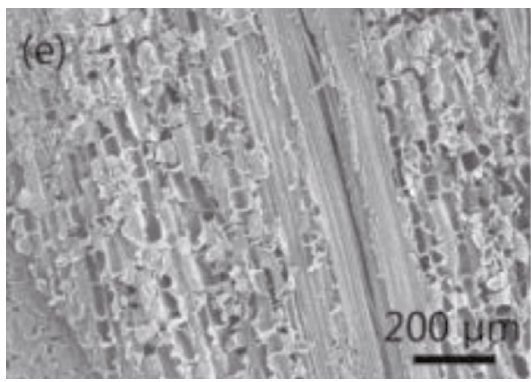

(e)

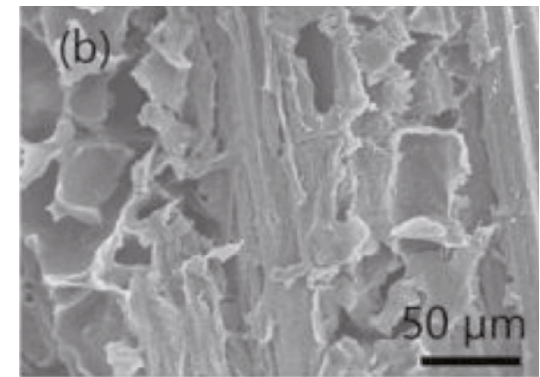

(b)

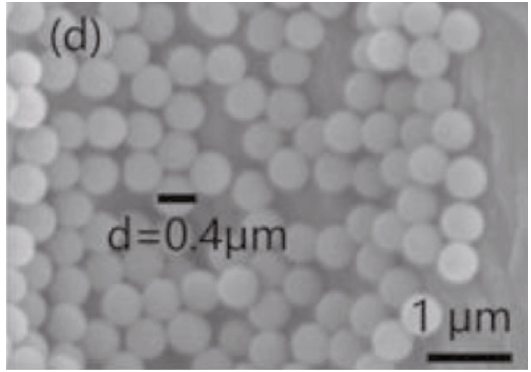

(d)

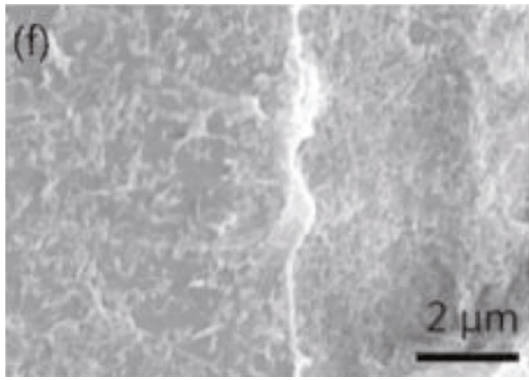

(f)

Figure 1: Typical low- and high-magnification SEM images of BT (a), RGO@SiO ${ }_{2} \mathrm{BT}$ (b, c, and d), and FAS-RGO@SiO $\mathrm{BT}$ (e, f).

\section{Results and Discussion}

3.1. SEM Analysis. Figure 1 shows the typical low- and highmagnification SEM images of BT and $\mathrm{RGO} @ \mathrm{SiO}_{2} \mathrm{BT}$. A large number of silica nanoparticles are grown on the surface of the bamboo material under low magnification. The silica nanoparticles are densely distributed on the surface layer of reduced graphene oxide. In high magnification, it is clear that the silicon dioxide is spherical with a diameter of $0.4 \mu \mathrm{m}$. It can be observed that the surface roughness increases after silica nanoparticle loading. After the hydrophobic treatment of the FAS-17, as shown in Figures 1(e) and 1(f), the rough spherical surface is covered with a waxy material. The surface presents various mastoid structures similar to the lotus leaf wax structure of papillae [16].

3.2. EDX Analysis. Figure 2 shows the chemical elements and element content of the three samples including BT, RGOBT, and RGO@SiO 2 BT. Elements of $\mathrm{C}, \mathrm{O}$, and $\mathrm{Au}$ can be detected in BT and RGOBT. Au originated from surface spraying of the three samples. The carbon and oxygen signals originated from the bamboo substrate and RGO coating [17]. The presence of $\mathrm{Si}$ in $\mathrm{RGO} @ \mathrm{SiO}_{2} \mathrm{BT}$ indicates that the silicon is successfully deposited on the surface of BT. Combined with the SEM analysis, it can be proved that the spherical silica nanoparticle is loading successfully on the RGOBT sample. Thus, the Si element could be detected as shown in Figure 2(c). Due to the silica particle coating, the element contents of $\mathrm{Si}$ and O on RGO@ $\mathrm{TiO}_{2} \mathrm{BT}$ increased when compared with those of RGOBT.

3.3. XRD Analysis. Figure 3 shows the XRD patterns of $\mathrm{BT}$, RGOBT, and RGO@SiO ${ }_{2} B T$. The typical diffraction peaks of cellulose from BT and RGOBT at $2 \theta=16.5^{\circ}$ and $22.5^{\circ}$ were observed [18]. After the hydrothermal treatment of graphene oxide dispersion, the characteristic diffraction peak of the graphene oxide disappeared. These prove that graphene oxide absorbed on the bamboo surface was reduced. The RGOBT was loaded with silica nanoparticles [19]. No new characteristic diffraction peaks were observed. It is found that the silica crystal loaded on the BT surface is an amorphous structure.

3.4. FTIR Analysis. Figure 4 presents the FTIR spectrum of $\mathrm{BT}, \mathrm{RGOBT}$, and RGO@SiO${ }_{2} \mathrm{BT}$. In the process of graphene oxide reduction, the absorption peaks of hydroxyl absorption were shifted to a high band of $3335 \mathrm{~cm}^{-1}$. Parts of the oxygen- 


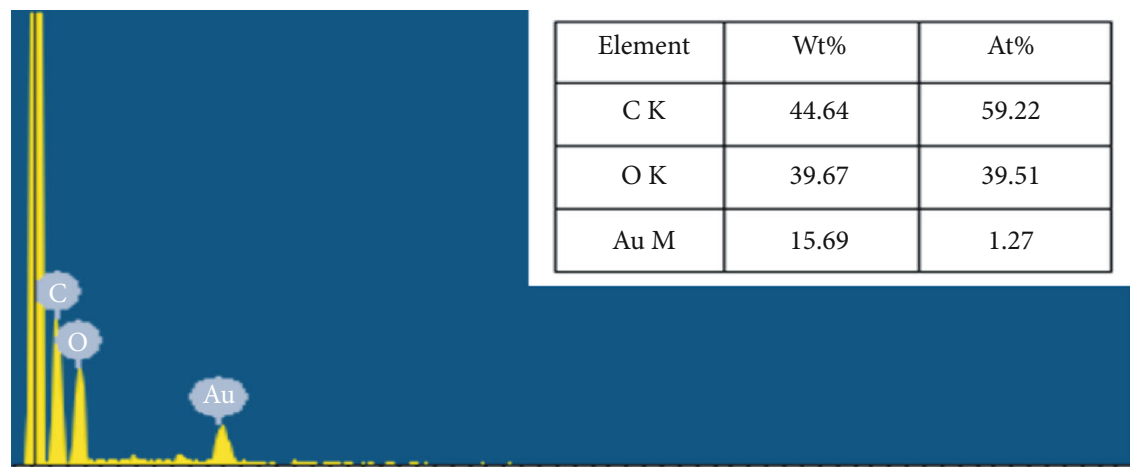

(a)

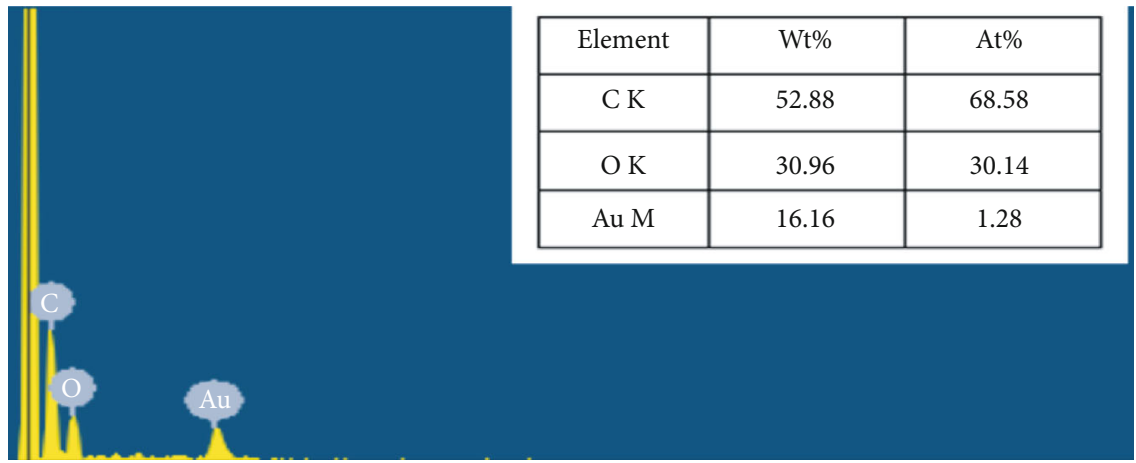

(b)

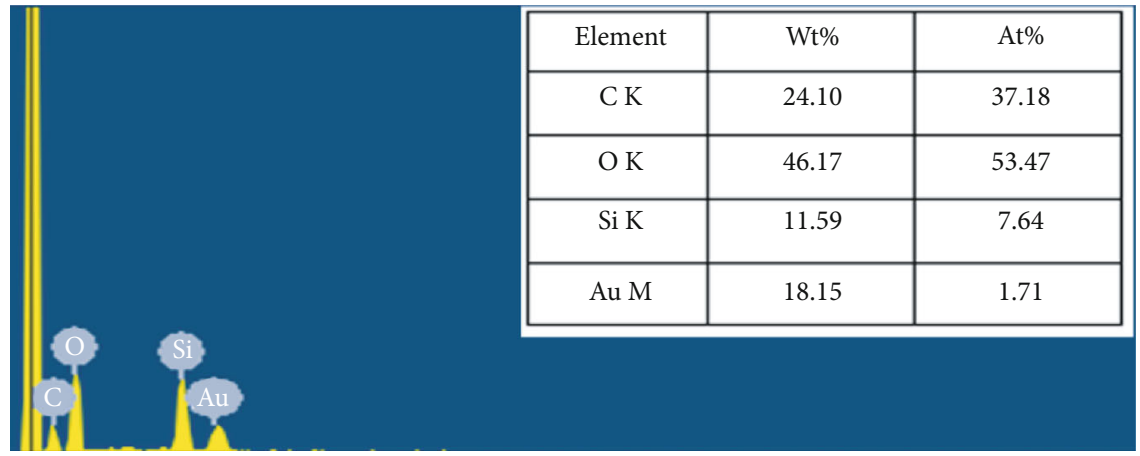

(c)

FIgURE 2: EDX spectra and element contents of BT (a), RGOBT (b), and RGO@SiO 2 BT (c).

containing functional groups are restored, resulting in fewer hydroxyl groups [20]. On the contrary, the formation of a $\mathrm{C}-\mathrm{O}$ bond between the hydroxyl group and graphene oxide on the surface of bamboo reduces the number of hydroxyl groups. The number of hydroxyl groups decreases as more silica nanospheres were deposited on the bamboo surface. This is consistent with the hydrophobic effect in the later waterproof test. The new absorption peak of the RGO@$\mathrm{SiO}_{2} \mathrm{BT}$ at $1644 \mathrm{~cm}^{-1}$ is the vibrational absorption peak of the graphene carbon skeleton. The absorption peak at $1104 \mathrm{~cm}^{-1}$ is the characteristic absorption peak of Si-O-Si. The silica nanoparticles were bonded to the reduced graphene network by a C-O bond during the hydrothermal process and then accumulated to form a nanosilica sphere layer.

3.5. XPS Analysis. To further characterize the elements and valence states of the samples, XPS analysis was performed.
Figure 5 is the XPS diagram of BT, RGOBT, and RGO@$\mathrm{SiO}_{2} \mathrm{BT}$. The peaks at $285 \mathrm{EV}$ can be regarded as $\mathrm{C} 1 \mathrm{~s}$. The peaks at $398 \mathrm{EV}$ can be regarded as $\mathrm{N}$ 1s. The peaks at 539 $\mathrm{EV}$ can be regarded as $\mathrm{O} 1 \mathrm{~s}$. The typical absorption peaks of $\mathrm{C}, \mathrm{N}$, and $\mathrm{O}$ were detected in the BT, RGOBT, and RGO@$\mathrm{SiO}_{2} \mathrm{BT}$ samples. These samples all contain these three elements, and their main valence states are the same. The peaks at 109 and $150 \mathrm{EV}$ can be regarded as Si 2p and Si 2s [21]. However, the Si typical absorption peaks were detected only in RGO@SiO $2 \mathrm{BT}$. The existence of $\mathrm{Si}^{4+}$ was confirmed by the Si 2p peak, and the existence of silica in $\mathrm{RGO@SiO} 2 \mathrm{BT}$ was confirmed.

3.6. Analysis of the Forming Mechanism. The formation mechanism of RGO@SiO $\mathrm{S}_{2} \mathrm{BT}$ is shown in Figure 6. The GO solution was first absorbed by the original BT and reduced to an RGO coating on the bamboo surface in the first 


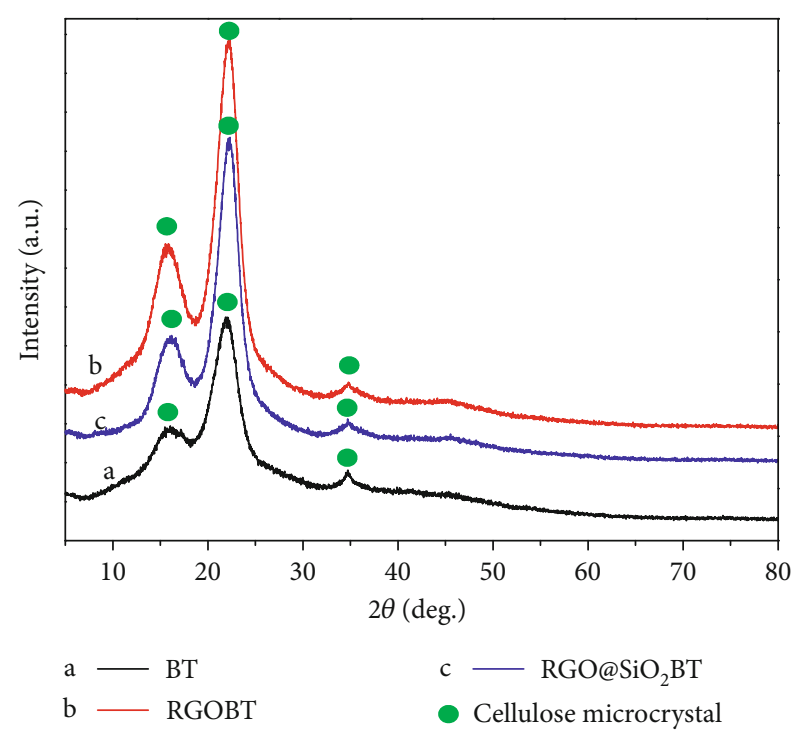

Figure 3: XRD pattern of BT (a), RGOBT (b), and RGO@SiO ${ }_{2} \mathrm{BT}$ (c).

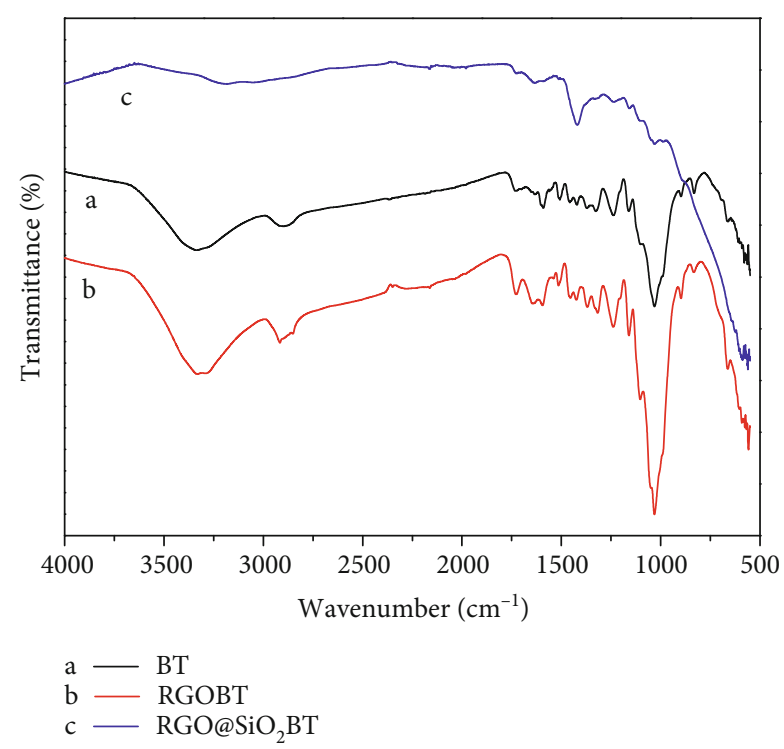

FIGURE 4: FTIR spectra of BT (a), RGOBT (b), and RGO@SiO ${ }_{2}$ BT (c).

hydrothermal process. This continuous surface of the assembled GO was formed on the bamboo block and was a reduction to RGOBT with a graphene network. The multiple layers of the RGO sheet were assembled onto the bamboo surface via hydrogen bonding and physical adsorption. As the hydrothermal reaction goes on in the second hydrothermal step, the silica nanoparticles were formed from the TEOS system and were deposited on the surface by the graphene reticular structure [22]. The graphene network provides more binding sites than bamboo, indicating that more silica nanospheres were deposited on the surface. The dense silica crystal layer is formed on the RGO@SiO 2 BT surface. Finally, to reduce the surface energy of $\mathrm{RGO} @ \mathrm{SiO}_{2} \mathrm{BT}$, the FAS modifi-

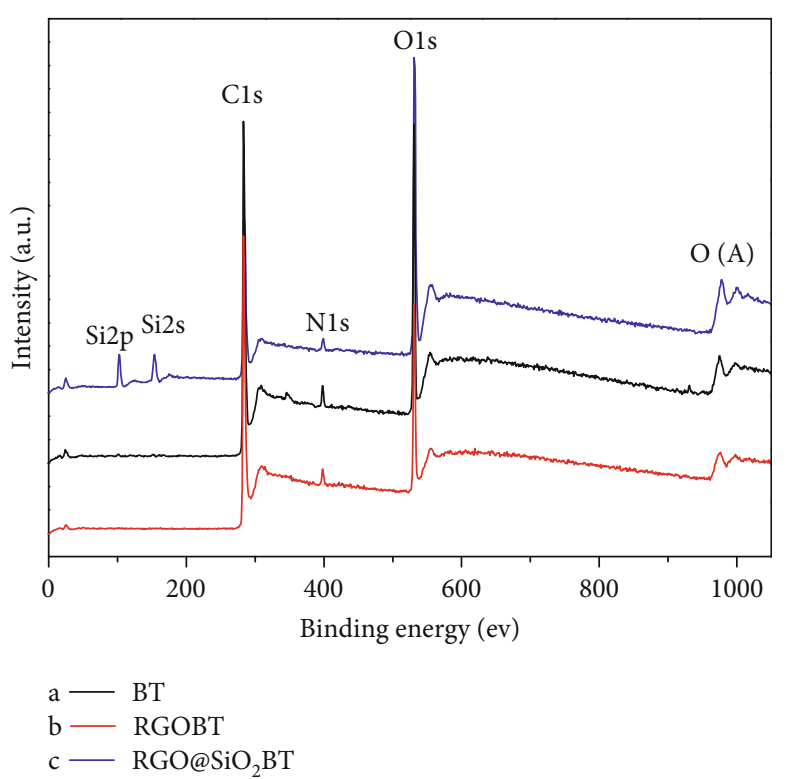

Figure 5: XPS spectra of BT (a), RGOBT (b), and RGO@SiO ${ }_{2} \mathrm{BT}$ (c).

cation layer on the surface was obtained via $\mathrm{Si}-\mathrm{OH}$ through the combination of hydrogen and silica nanospheres.

3.7. Wettability Analysis. Figure 7 is the water contact angle (WCA) image of BT, RGOBT, RGO@SiO 2 BT, and FAS$\mathrm{RGO} @ \mathrm{SiO}_{2} \mathrm{BT}$ samples. The WCA of the BT sample is $19^{\circ}$ in Figure 7(a). Bamboo is a hydrophilic matrix, and its WCA value is low [23]. Through the loading of reduction of graphene oxide, the RGOBT surface becomes more hydrophilic, and its WCA is $0^{\circ}$, as shown in Figure 7(b). One of the possible reasons is speculated that graphene oxide still exists on the bamboo surface with carboxyl, hydroxyl, and other oxygen-containing groups. Another possible reason is that reduced graphene oxide nanosheets deposited on the surface are not uniform. The intermediate layer-by-layer stacking has pores resulting in the infiltration of water droplets with improved hydrophilicity [24]. Figure 7(c) shows that the WCA of RGO@SiO 2 BT reaches $140^{\circ}$, which proves that RGO@SiO 2 BT has higher hydrophobic properties. According to the SEM analysis, silica nanospheres were loaded over the entire surface of the RGO network. The hydrophobicity of the BT surface with micro- and nanoscale protuberance can be significantly improved. However, the WCA still did not meet the requirements of superhydrophobicity with a WCA above $150^{\circ}$.

Jiang et al. studied the causes of Aquarium paludum Fabricius walking on the water surface and found that the two scales with micro- and nanostructures show superhydrophobicity $[25,26]$. The self-cleaning and superhydrophobic materials were prepared by bionically designing a similar structure with the lotus leaf and the Aquarium paludum Fabricius. It is found that the hierarchical structure of nanometer and micron structure can effectively reduce the rolling angle of water droplets on the surface, which directly affects the movement trend of water droplets. Further, by the FAS-17 

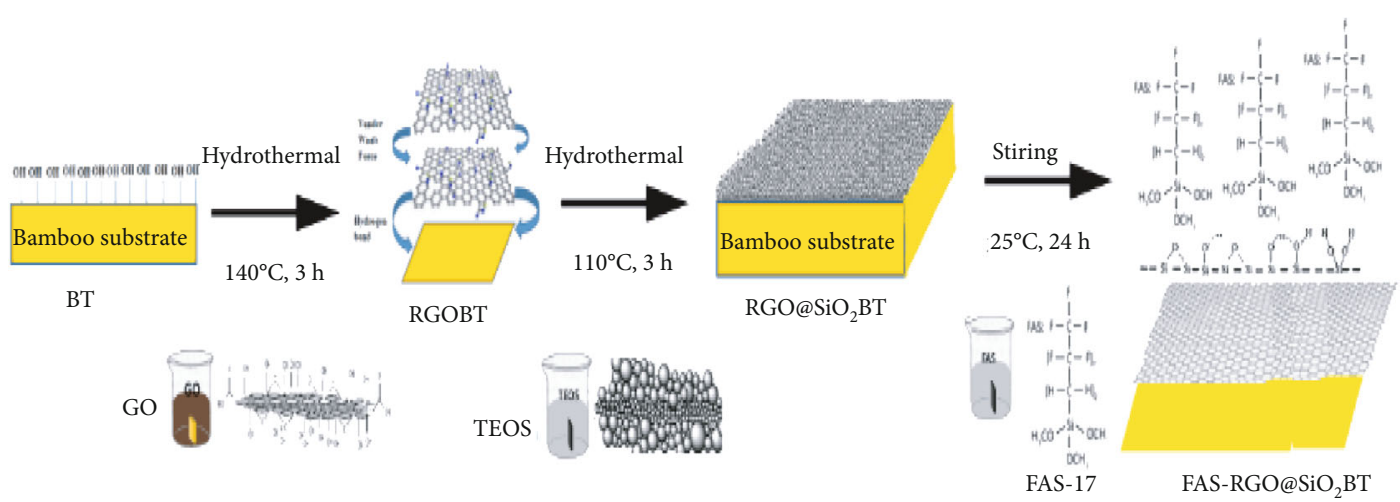

Figure 6: Schematic illustration and synthetic mechanism of FAS-RGO@SiO 2 BT.

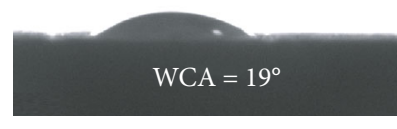

(a)

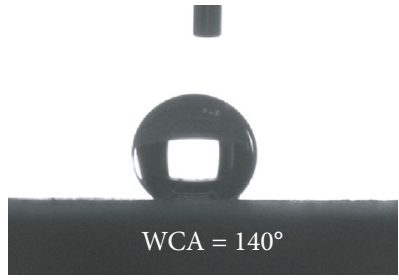

(c)

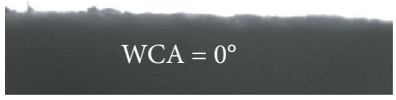

(b)

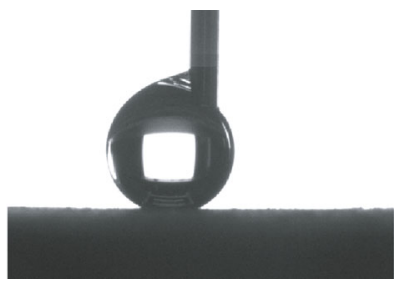

(d)

Figure 7: WCA of (a) BT, (b) RGOBT, (c) RGO@SiO ${ }_{2} \mathrm{BT}$, and (d) FAS-RGO@SiO ${ }_{2} \mathrm{BT}$.

modification, the surface free energy of FAS-RGO@SiO, BT goes down. When $5 \mu \mathrm{l}$ of water droplet was close to the surface of FAS-RGO@SiO 2 BT, the droplet still does not spread to the surface and always maintains the circular shape. Until after the bamboo is away from the needle, the liquid is still hanging on the needles, which is shown in Figure $7(\mathrm{~d})$. The WCA of FAS-RGO@SiO ${ }_{2} \mathrm{BT}$ shows an inaccurate value; however, it is far more than $150^{\circ}$. It is proven that the FASRGO@SiO 2 BT has a good hydrophobic effect.

Typically, bamboo products have good hydrophilicity and can easily absorb water. The hydrophilicity of bamboo leads to deformation, crack, and mold, among others, thus affecting its application. It is very interesting to achieve the conversion of a bamboo surface from hydrophilicity to hydrophobicity. Figure 8 is the hydrophobic effect of BT, RGOBT, RGO@SiO 2 BT, and FAS-RGO@SiO 2 BT in contact with several liquids.

By comparison, it was found that BT and RGOBT had no hydrophobic effect on the eight types of liquid. Both the RGO@SiO 2 BT and FAS-RGO@SiO 2 BT have a hydrolysis effect on tea, orange juice, milk, coffee, blue ink, red wine, and green juice. However, the FAS-RGO@SiO $2 \mathrm{BT}$ is superior to $\mathrm{RGO} @ \mathrm{SiO}_{2} \mathrm{BT}$, which is consistent with the test results of
WCA. In addition, both the RGO@SiO 2 BT and FAS-RGO@$\mathrm{SiO}_{2} \mathrm{BT}$ are lipophilic through the results of contact with oil. The RGO@SiO 2 BT and FAS-RGO@SiO 2 BT were not hydrophobic on oil. Thus, it is necessary to further study the superhydrophobicity to oil.

Figure 9 is the digital photos of FAS-RGO@SiO ${ }_{2} \mathrm{BT}$ in contact with various $\mathrm{pH}$ solutions. The FAS-RGO@ $\mathrm{SiO}_{2} \mathrm{BT}$ has an obvious hydrophobic effect on any of the various solutions with a $\mathrm{pH}$ of 1-14. It can be intuitively observed that the preparation of FAS-RGO@SiO 2 BT still maintains a good hydrophobic effect on the strong acid or strong alkali solution. As a result, the FAS-RGO@SiO $2 \mathrm{BT}$, to be developed as an outdoor material, will have good hydrophobic characteristics and can resist acid rain.

3.8. Dimensional Stability Analysis. The BT and FAS-RGO@$\mathrm{SiO}_{2} \mathrm{BT}$ samples were immersed in water separately. Because the density of the bamboo material used in this experiment is $\sim 0.4 \mathrm{~g} / \mathrm{cm}^{3}$, it is significantly less than the density of water, so the two samples will float on the water surface. Without considering pore and water absorption, in the formula of buoyancy theory, the bamboo block should be immersed in water with a thickness of $40 \%$. The FAS-RGO@SiO 2 BT in 




(a)

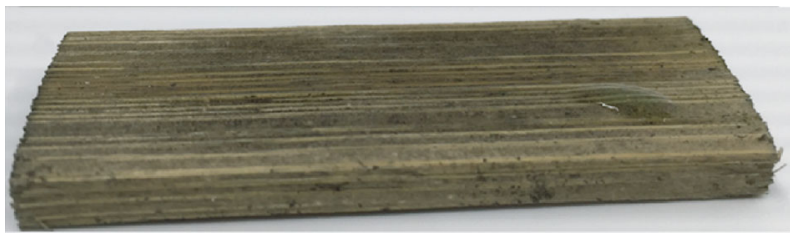

(c)

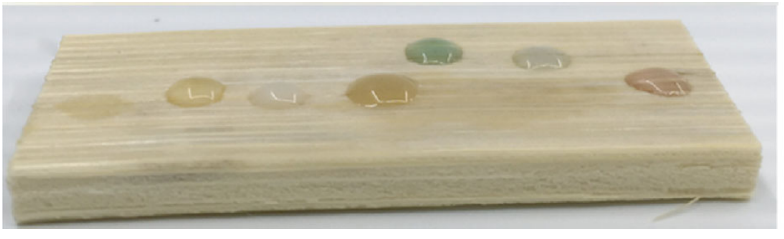

(b)



(d)

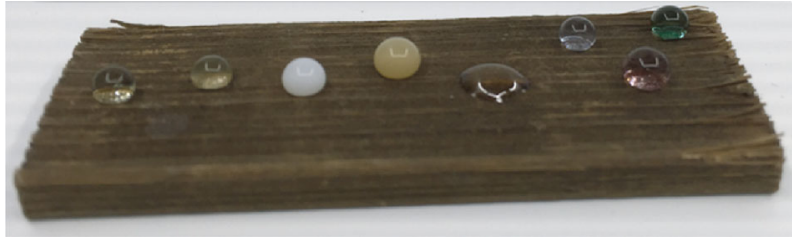

(e)

Figure 8: Digital photos of (b) BT, (c) RGOBT, (d) RGO@SiO ${ }_{2}$ BT, and (e) FAS-RGO@SiO 2 BT in contact with the (a) eight common liquids in daily life.

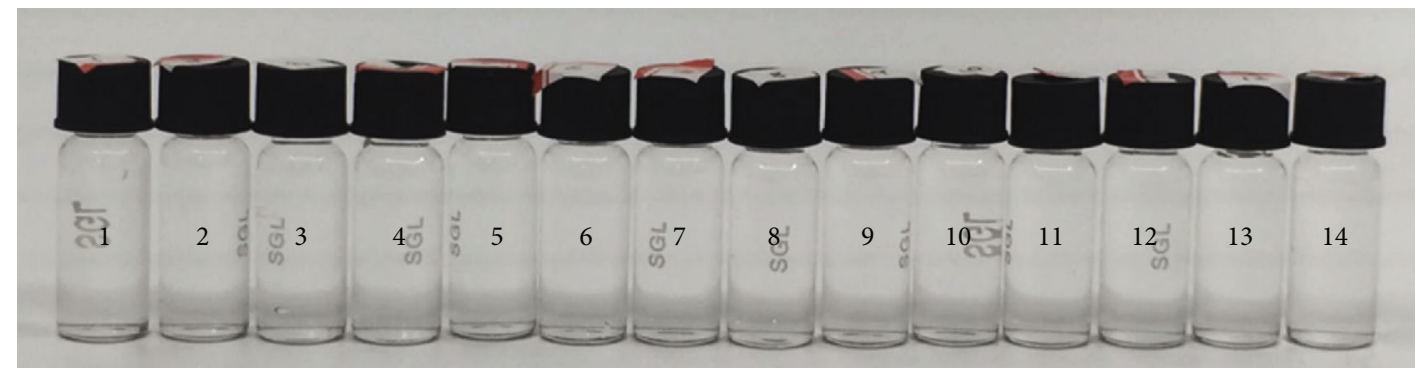

(a)

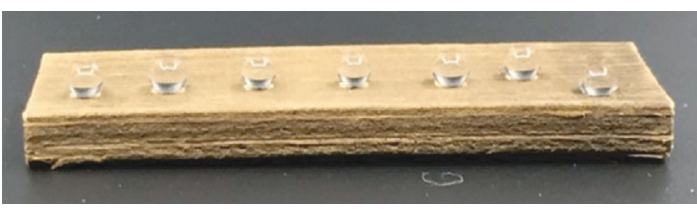

(b)

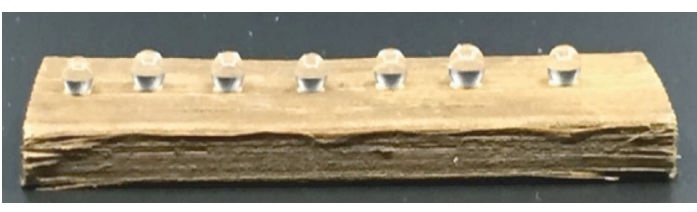

(c)

Figure 9: Digital photos of FAS-RGO@SiO 2 BT in contact with solution of $\mathrm{pH} 1$ to 14.

Figure 10(a) has been dipped into the water due to its weight (actually less than 40\%). FAS-RGO@SiO 2 BT does not absorb water, and only the bottom surface adheres to the water surface. The material is significantly larger than FAS-RGO@$\mathrm{SiO}_{2} \mathrm{BT}$ due to its gravity and moisture absorption. In Figure 10(b), both samples were immersed in water by external forces. Without the external forces, the bamboo will quickly pop out of the water, as shown in Figure 10(a). FAS-RGO@SiO 2 BT samples were still kept above the surfaces and were not moist. As shown in Figures 10(c) and $10(\mathrm{~d})$, if water drops were added to the bamboo blocks, the surface was all saturated with water, while the surface water beads of the FAS-RGO@SiO ${ }_{2} \mathrm{BT}$ maintained a high WCA. With the external forces, BT and FAS-RGO@SiO 2 BT were immersed in water at room temperature, and the measurement and analysis of water absorption weight and thickness were repeated regularly.

The weight and thickness of BT and FAS-RGO@SiO 2 BT were measured for a long time. And we tested the water absorption rate and thickness swelling rate of BT and FAS$\mathrm{RGO} @ \mathrm{SiO}_{2} \mathrm{BT}$ at room temperature for 90 days. As the results shown in Figure 11, the BT samples absorb more 


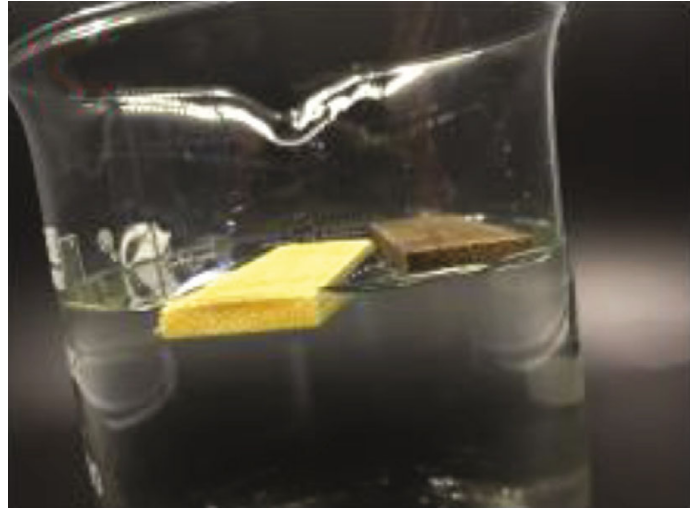

(a)

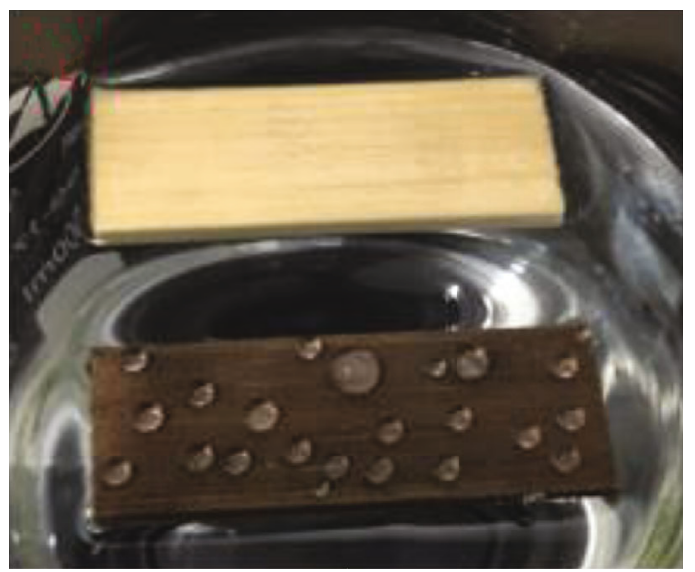

(c)

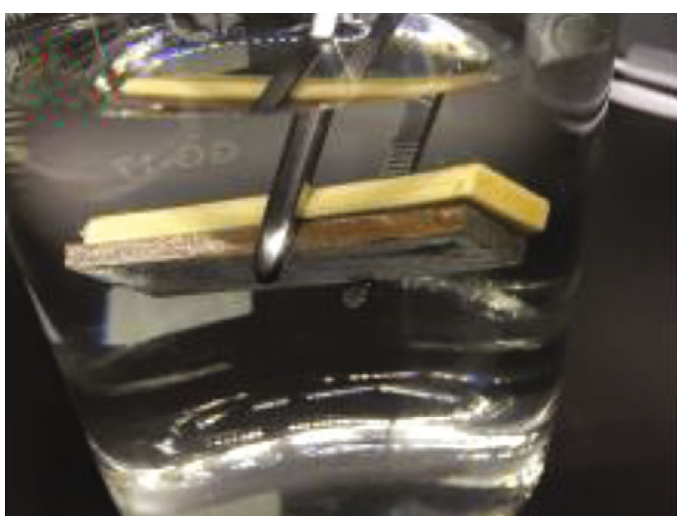

(b)

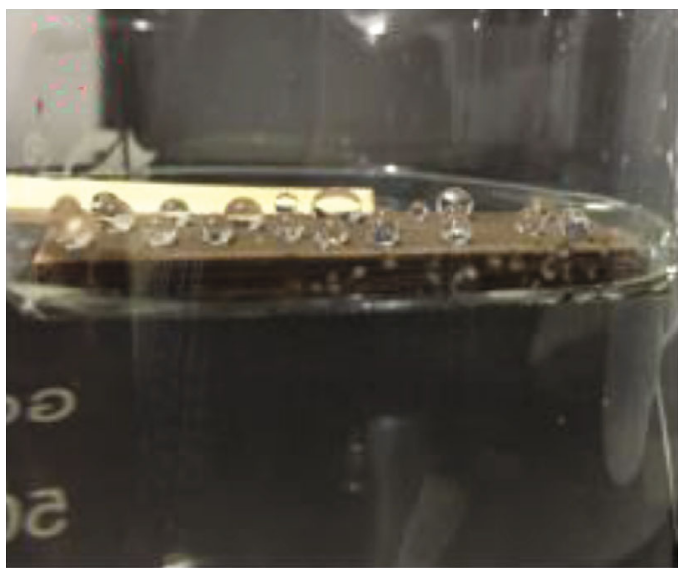

(d)

FIGURE 10: Digital photos of immersion of BT and FAS-RGO@SiO ${ }_{2} \mathrm{BT}$ into water.
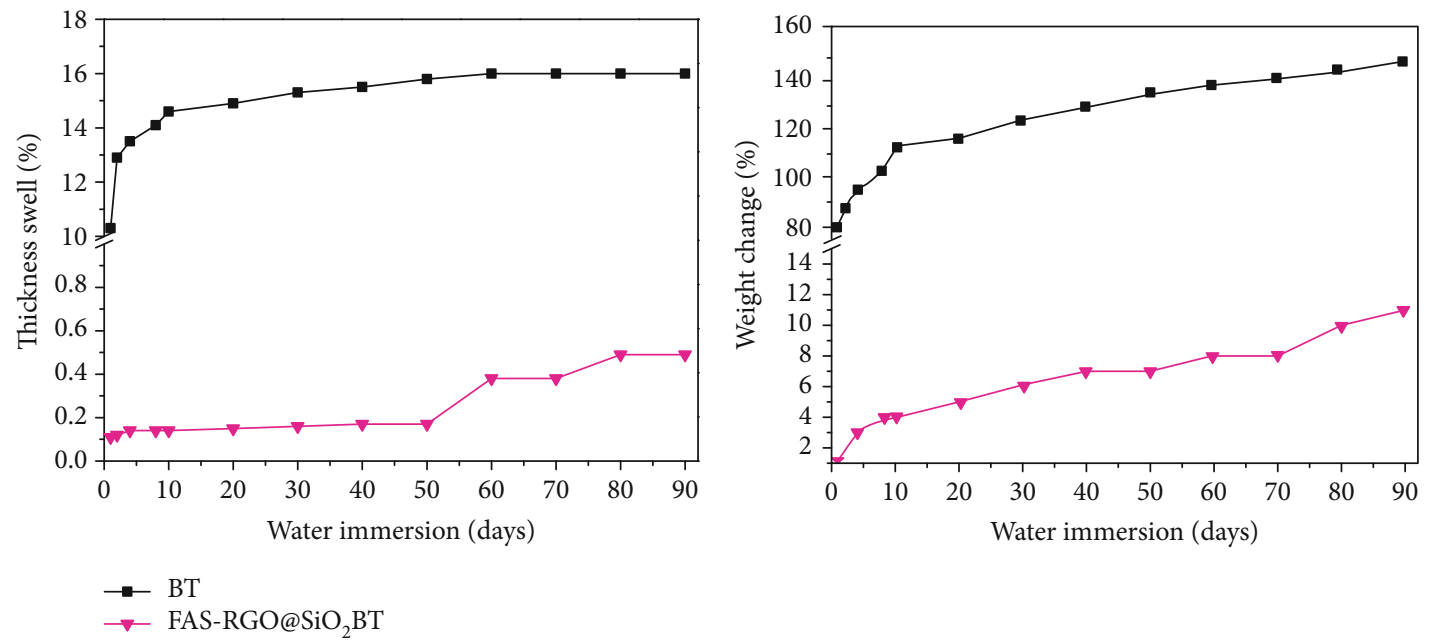

FIGURE 11: Water absorption and thickness swelling rate of BT and FAS-RGO@SiO 2 BT immersion in water for 90 days.

water in a short period and expand to a higher thickness. However, the water absorption and weight change of FASRGO@SiO ${ }_{2} \mathrm{BT}$ are much lower than those of BT. Under the same treatment time, the thickness swelling rate of FASRGO@SiO $\mathrm{BT}_{2} \mathrm{BT}$ also much smaller than that of BT. After a 90-day test, the water absorption of BT samples increased to $145 \%$, and the thickness swelling rate reaches $16.3 \%$.

\section{Conclusions}

The RGO@SiO ${ }_{2} \mathrm{BT}$ samples have been successfully prepared by the two-step hydrothermal method. The samples were analyzed using SEM, EDX, XRD, FTIR, and XPS. The results showed that the reduced graphene oxide and silica nanosphere were combined by a C-O bond. The silica on the 
bamboo surface is amorphous. The diameter of the silica nanosphere is $\sim 0.4 \mu \mathrm{m}$, and the surface roughness of RGO@$\mathrm{SiO}_{2} \mathrm{BT}$ increases. The formation mechanism of FASRGO@SiO 2 BT was proposed: it can be expressed as follows: the graphene oxide is reduced and deposited on the bamboo surface, and the RGOBT is obtained by self-assembly layer accumulation. In the TEOS colloidal solution, the silica nanoparticles are accumulating on the surface of RGOBT. Finally, the silica nanosphere crystal with a diameter of $0.4 \mu \mathrm{m}$ is formed in the outermost layer. The FAS modification layer was obtained by impregnating the methanol solution in FAS-17. The surface energy of the RGO@SiO $2 \mathrm{BT}$ was reduced. The interface is combined with the hydrogen bond and silica nanomaterial.

The FAS-RGO@SiO 2 BT bamboo has excellent hydrophobic properties, such as the tea, orange juice, milk, coffee, blue ink, red wine, and green juice liquid. Simultaneously, the FAS-RGO@SiO 2 BT has an excellent hydrophobic effect on the pH 1-14 solution. The FAS-RGO @SiO 2 BT bamboo can be used as an outdoor material and has the application prospect in resisting acid rain. The RGO@TiO 2 BT has excellent dimensional stability. After the 90-day bubble water test, for the BT samples, the absorption increased to $145 \%$ and the thickness expanded to 16.3\%; however, for the FAS-RGO@$\mathrm{SiO}_{2} \mathrm{BT}$ samples, the water absorption rate is $9.7 \%$ and the thickness swelling rate is $0.5 \%$. The wettability and dimensional stability of the FAS-RGO@SiO $2 \mathrm{BT}$ significantly improved compared to those of BT.

\section{Data Availability}

The data used to support the findings of this study are available from the corresponding author upon request.

\section{Conflicts of Interest}

The authors declare no conflict of interest.

\section{Acknowledgments}

The authors would like to thank the Cooperation Project of Zhejiang Province Project of Scientific Research Institutes (Grant no. 2021F1065-7), Zhejiang Province \& China Forestry Academy (Grant no. 2014SY13), and National Natural Science Foundation of China (Grant no. 31470586) for the financial support.

\section{References}

[1] X. Q. Wang and H. Q. Ren, "Comparative study of the photodiscoloration of moso bamboo (Phyllostachys pubescens Mazel) and two wood species," Applied Surface Science, vol. 254, no. 21, pp. 7029-7034, 2008.

[2] P. K. Kushwaha and R. Kumar, "Influence of chemical treatments on the mechanical and water absorption properties of bamboo fiber composites," Journal of Reinforced Plastics and Composites, vol. 30, no. 1, pp. 73-85, 2011.

[3] K. Seo, M. Kim, and D. H. Kim, "Candle-based process for creating a stable superhydrophobic surface," Carbon, vol. 68, pp. 583-596, 2014.
[4] S. Wang, C. Liu, G. Liu, M. Zhang, J. Li, and C. Wang, "Fabrication of superhydrophobic wood surface by a sol-gel process," Applied Surface Science, vol. 258, no. 2, pp. 806-810, 2011.

[5] S. Wang, C. Wang, C. Liu, M. Zhang, H. Ma, and J. Li, "Fabrication of superhydrophobic spherical-like $\alpha$-FeOOH films on the wood surface by a hydrothermal method," Colloids and Surfaces A: Physicochemical and Engineering Aspects, vol. 403, pp. 29-34, 2012.

[6] L. Gao, Y. Lu, J. Cao, J. Li, and Q. Sun, "Reversible photocontrol of wood-surface wettability between superhydrophilicity and superhydrophobicity based on a $\mathrm{TiO}_{2}$ film," Journal of Wood Chemistry and Technology, vol. 35, no. 5, pp. 365-373, 2015.

[7] L. Gao, S. Xiao, W. Gan, X. Zhan, and J. Li, "Durable superamphiphobic wood surfaces from $\mathrm{Cu}_{2} \mathrm{O}$ film modified with fluorinated alkyl silane," RSC Advances, vol. 5, no. 119, pp. 9820398208, 2015.

[8] W. Gan, L. Gao, W. Zhang, J. Li, and X. Zhan, "Fabrication of microwave absorbing $\mathrm{CoFe}_{2} \mathrm{O}_{4}$ coatings with robust superhydrophobicity on natural wood surfaces," Ceramics International, vol. 42, no. 11, pp. 13199-13206, 2016.

[9] B. Hui, G. Li, J. Li, and B. K. Via, "Hydrothermal deposition and photoresponsive properties of $\mathrm{WO}_{3}$ thin films on wood surfaces using ethanol as an assistant agent," Journal of the Taiwan Institute of Chemical Engineers, vol. 64, pp. 336-342, 2016.

[10] A. Lozhechnikova, H. Bellanger, B. Michen, I. Burgert, and M. Österberg, "Surfactant-free carnauba wax dispersion and its use for layer-by-layer assembled protective surface coatings on wood," Applied Surface Science, vol. 396, pp. 1273-1281, 2017.

[11] J. Li, Y. Chen, J. Xu et al., "The influence of media treatments on color changes, dimensional stability, and cracking behavior of bamboo scrimber," International Journal of Polymer Science, vol. 2018, Article ID 6068589, 7 pages, 2018.

[12] S. Huang, Y. Zhang, J. Shi, and W. Huang, "Superhydrophobic particles derived from nature-inspired polyphenol chemistry for liquid marble formation and oil spills treatment," ACS Sustainable Chemistry \& Engineering, vol. 4, no. 3, pp. 676-681, 2016.

[13] Y. Levi-Kalisman, G. Falini, L. Addadi, and S. Weiner, "Structure of the nacreous organic matrix of a bivalve mollusk shell examined in the hydrated state using cryo-TEM," Journal of Structural Biology, vol. 135, no. 1, pp. 8-17, 2011.

[14] J. Wang, Q. Yao, M. Sheng, C. Jin, and Q. Sun, “One-step preparation of graphene oxide/cellulose nanofibril hybrid aerogel for adsorptive removal of four kinds of antibiotics," Journal of Nanomaterials, vol. 2017, Article ID 5150613, 10 pages, 2017.

[15] General Administration of Quality Supervision, Inspection and Quarantine of the People's Republic of China, "GB/T 1928-2009 General Rules of Wood Physical and Mechanical Test Methods," Beijing: Standards Press of China, 2009.

[16] H. J. Ensikat, P. Ditsche-Kuru, C. Neinhuis, and W. Barthlott, "Superhydrophobicity in perfection: the outstanding properties of the lotus leaf," Beilstein Journal of Nanotechnology, vol. 2, no. 1, pp. 152-161, 2011.

[17] J. Wang, Q. Sun, F. Sun, Q. Zhang, and C. Jin, "Layer-by-layer self-assembly of reduced graphene oxide on bamboo timber surface with improved decay resistance," European Journal of Wood and Wood Products, vol. 76, no. 4, pp. 1223-1231, 2018. 
[18] C. Jin, J. Li, S. Han, J. Wang, and Q. Sun, "A durable, superhydrophobic, superoleophobic and corrosion-resistant coating with rose-like $\mathrm{ZnO}$ nanoflowers on a bamboo surface," Applied Surface Science, vol. 320, pp. 322-327, 2014.

[19] J. Wang, C. Jin, Q. Sun, and Q. Zhang, "Fabrication of nanocrystalline anatase $\mathrm{TiO}_{2}$ in a graphene network as a bamboo coating material with enhanced photocatalytic activity and fire resistance," Journal of Alloys and Compounds, vol. 702, pp. 418-426, 2017.

[20] B. F. Tjeerdsma and H. Militz, "Chemical changes in hydrothermal treated wood: FTIR analysis of combined hydrothermal and dry heat-treated wood," Holz als Roh- und Werkstoff, vol. 63, no. 2, pp. 102-111, 2005.

[21] M. C. Biesinger, L. W. M. Lau, A. R. Gerson, and R. S. C. Smart, "Resolving surface chemical states in XPS analysis of first row transition metals, oxides and hydroxides: $\mathrm{Sc}, \mathrm{Ti}, \mathrm{V}, \mathrm{Cu}$ and Zn," Applied Surface Science, vol. 257, no. 3, pp. 887-898, 2010.

[22] O. C. Compton and S. B. T. Nguyen, "Graphene oxide, highly reduced graphene oxide, and graphene: versatile building blocks for carbon-based materials," Small, vol. 6, no. 6, pp. 711-723, 2010.

[23] S. Strand, D. C. Standnes, and T. Austad, "New wettability test for chalk based on chromatographic separation of $\mathrm{SCN}^{-}$and $\mathrm{SO}_{4}{ }^{2-}$," Journal of Petroleum Science \& Engineering, vol. 52, no. 1-4, pp. 187-197, 2006.

[24] J. Li, Q. Sun, B. Fan, H. Zheng, C. Yan, and C. Jin, "Fabrication of biomimetic superhydrophobic plate-like $\mathrm{CaCO}_{3}$ coating on the surface of bamboo timber inspired from the biomineralization of nacre in seawater," Nano Reports, vol. 1, pp. 9-14, 2015.

[25] X. F. Gao and L. Jiang, "Water-repellent legs of water striders," Nature, vol. 432, no. 7013, p. 36, 2004.

[26] L. Jiang, R. Wang, B. Yang et al., "Binary cooperative complementary nanoscale interfacial materials," Pure and Applied Chemistry, vol. 72, no. 1-2, pp. 73-81, 2000. 